\title{
Doença crítica crônica: artigo de revisão narrativa
}

\section{Chronic critical Illness: narrative review article}

\author{
Sarah Musy Leitão ${ }^{1}$, Penélope Matos Wirtzbiki², Otílio José Nicolau de Oliveira ${ }^{3,4}$
}

1. Programa de Residência Médica em Geriatria pela Escola de Saúde Pública do Ceará (ESP/CE) /Hospital Universitário Walter Cantídio (HUWC), Fortaleza, CE, Brasil. 2. Comissão de Cuidados Paliativos do Hospital Geral de Fortaleza (HGF), Fortaleza, CE, Brasil. 3. Serviço de Clínica médica do Hospital Geral de Fortaleza (HGF), Fortaleza, CE, Brasil. 4. Hospital Universitário Walter Cantídio (HUWC), Fortaleza, CE, Brasil.

\begin{abstract}
Resumo
Introdução: Existem três desfechos possíveis para o paciente crítico: controle rápido da instabilidade com recuperação funcional; óbito; curso subagudo, no qual há sobrevivência com persistência de disfunções orgânicas e dependência prolongada de suporte de manutenção da vida. A última possibilidade é a doença crítica crônica: condição devastadora, com alta taxa de mortalidade, porém desconhecida de muitos médicos e familiares. Objetivo: tornar o tema doença crítica crônica mais acessível aos médicos, por meio de revisão da literatura. Método: Revisão de literatura narrativa. Foram selecionados artigos publicados nos últimos 10 anos, de 2007 a 2017, localizados pelos descritores: ventilação mecânica/estado crítico/doença crônica, e suas traduções para inglês (mechanical ventilation, critical condition, chronic disease), nas bases de dados Bireme e SciELO. Resultados: Foram revisados 70 artigos. Foram apresentados definição, epidemiologia, fisiopatologia, quadro clínico, tratamento e prognóstico da doença crítica crônica. Conclusão: A doença crítica crônica tem grande importância clínica e epidemiológica, com impacto no sistema de saúde. Os médicos precisam conhecer melhor essa síndrome, diagnosticá-la e tratá-la adequadamente, inclusive iniciando a abordagem de cuidados paliativos o quanto antes.
\end{abstract}

Palavras-chave: Ventilação mecânica. Estado crítico. Doença crônica.

\begin{abstract}
Introduction: There are three possible outcomes for critically ill patients: rapid control of instability with functional recovery; death; subacute course, in which there is survival to the acute insult with persistent organ dysfunction and prolonged dependence on life-sustaining support. The last possibility is the chronic critical illness: a devastating condition with a high mortality rate, about which both physicians and family members have lack of knowledge. Objective: To make the subject chronic critical illness more accessible to health professionals, through a literature review. Method: Narrative Literature review. We have selected articles published in the last 10 years, from 2007 to 2017, located through the keywords: mechanical ventilation, critical condition, chronic disease, and their translations into Portuguese (ventilação mecânica, estado crítico, doença crônica), in Bireme and SciELO databases. Results: 70 articles were reviewed. Definitions, epidemiology, physiopathology, clinical manifestations, treatment and prognosis of chronic critical illness were presented. Conclusion: Chronic critical illness has great clinical and epidemiological importance, with impact on the health system. Physicians need to get to know this syndrome better, diagnose and treat it properly, including the beginning of palliative care approaches as soon as possible.
\end{abstract}

Key words: Mechanical ventilation. Critical condition. Chronic disease.

\section{INTRODUÇÃO}

Os avanços tecnológicos progressivos nos cuidados intensivos têm permitido que mais pacientes sobrevivam a condições críticas agudas. No entanto, muitos desses sobreviventes persistem com múltiplas disfunções orgânicas e dependência de cuidados intensivos prolongados, principalmente a ventilação mecânica, uma síndrome conhecida como doença crítica crônica. O termo foi criado por Girard e Raffin, em um artigo publicado em 1985 com o título "Salvar ou deixar morrer?" Os autores focaram em um subgrupo de pacientes que sobreviveram ao episódio inicial da doença crítica, mas permaneceram dependentes de cuidados intensivos, nem morrendo no período agudo dos cuidados intensivos, nem se recuperando ${ }^{1,2,3,4}$.

A doença crítica crônica é uma condição devastadora: a mortalidade excede à da maioria das malignidades e a dependência funcional persiste para a maioria dos sobreviventes.
Uma parcela importante dos pacientes tem baixa qualidade de vida, com grande sobrecarga emocional também para seus familiares e cuidadores ${ }^{1,2,3,4,5,6,7,8,9,10,11,12,13,14, ~ 15 . ~}$

Apesar de haver um consenso quanto à sua definição (pelo menos 21 dias em ventilação mecânica por pelo menos 6 horas/ dia), alguns estudos têm proposto critérios alternativos, como a indicação de traqueostomia, um período diferente de ventilação mecânica ou a admissão em uma unidade intermediária para desmame de ventilação mecânica. Independente da definição, a incidência da doença crítica crônica tem dobrado nas últimas décadas e ainda deverá crescer nos próximos anos ${ }^{3,4}$.

Em geral, a população dos pacientes críticos crônicos é caracterizada pela heterogeneidade, uma alta taxa de mortalidade e uma grande demanda por serviços de cuidados pós-agudos de reabilitação ou unidades de cuidados 
especiais $^{1,3,16,17,18,19}$.

Apesar da importância da doença crítica crônica e seu impacto na organização do sistema de saúde, ainda faltam dados acerca de seus aspectos epidemiológicos e desfecho dos pacientes, particularmente nas unidades de terapia intensiva brasileiras. Essas informações poderiam contribuir para melhor conhecer a dimensão da doença crítica crônica no Brasil e estimar a necessidade de leitos de cuidados pós-agudos de reabilitação ${ }^{16}$.

\section{MÉTODOS}

\section{Estratégia de busca e seleção}

O método adotado foi de revisão de literatura, consistindo na busca de artigos científicos das bases de dados SciELO e Bireme, em 29 de março de 2017. Os artigos foram selecionados a partir das seguintes palavras-chave: "ventilação mecânica" ou "estado crítico" ou "doença crônica", além de suas traduções para inglês: "mechanical ventilation" ou "critical condition" ou "chronic disease".Vale ressaltar que os termos "ventilação mecânica prolongada", "doença crítica crônica" e seus sinônimos ainda não constam da lista de descritores dos bancos de dados.

Na primeira etapa, foram considerados para inclusão na revisão: artigos originais nas línguas inglesa ou portuguesa, publicados nos últimos 10 anos, de 2007 a 2017, a respeito de humanos, excluídas as faixas etárias pediátricas. Há poucos dados na literatura sobre o quadro clínico, o prognóstico e o tratamento das crianças e dos adolescentes diagnosticados com doença crítica crônica, necessitando de mais trabalhos específicos acerca dessa população.

Figura 1. Estratégia de Busca e Seleção

\section{Extração de dados}

Os dados que foram extraídos dos estudos e incluídos nesta revisão são: definição, epidemiologia, fisiopatologia, quadro clínico, tratamento e prognóstico.

\section{RESULTADOS E DISCUSSÃO}

Encontraram-se 2606 artigos em SciELO e 20322 artigos em Bireme, totalizando 22.928 estudos. Na segunda etapa da busca, os títulos dos artigos foram avaliados a fim de determinar se eles eram potencialmente elegíveis para inclusão. 0 critério de inclusão foi conter em seu título informações sobre ventilação mecânica prolongada ou doença crítica crônica, resultando em 115 artigos. Após isso, foram excluídos os artigos duplicados ${ }^{5}$ e os remanescentes dos filtros: trabalhos que abordam temas pediátricos ${ }^{7}$, artigos de revisão ${ }^{23}$, editoriais ${ }^{5}$ e comentários ${ }^{5}$.

Ao final da seleção, restaram 70 artigos. Os textos completos deles foram analisados e utilizados na revisão. (Figura 1)

\section{Definição}

Apesar de ser uma síndrome complexa, a principal característica da doença crítica crônica (DCC) é a ventilação mecânica prolongada (VMP), cuja definição varia na literatura entre 2 dias e 4 semanas $^{3,4}$.

Existe um consenso (2004) quanto à definição de VMP: mais de 21 dias consecutivos em ventilação mecânica por mais de 6 horas diárias. Há, porém, definições alternativas, incluindo a necessidade de traqueostomia e utilizando durações diferentes

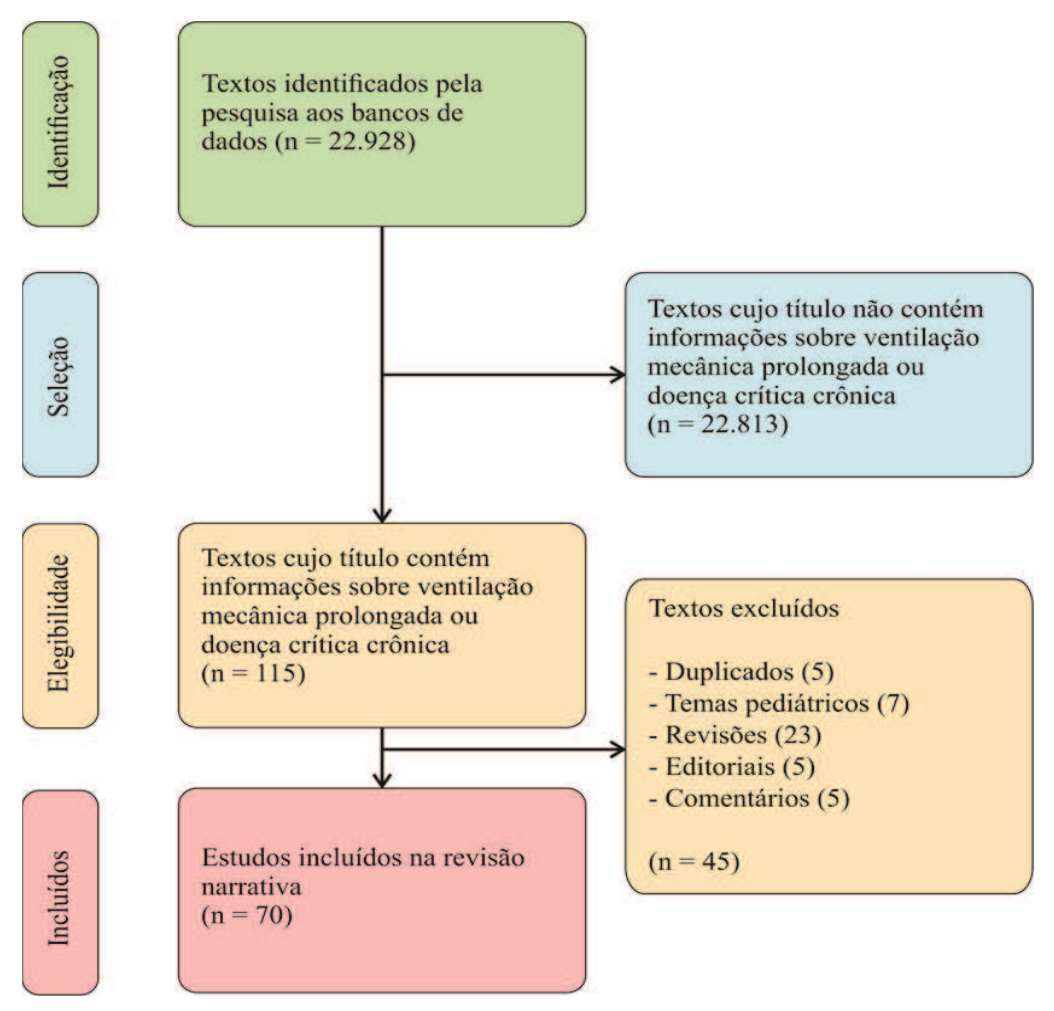


de ventilação mecânica (VM). A indicação de traqueostomia, excluindo indicações relacionadas à cirurgia de cabeça e pescoço, mostra que o médico está prevendo o curso de uma VMP e sobrevivência por um período de tempo razoável ${ }^{3,4,8}$.

A dificuldade de estabelecer a traqueostomia como definição de DCC se deve à variação do momento em que se indica o procedimento entre as unidades de terapia intensiva (UTIs) e entre os médicos de uma mesma UTI. Na literatura, não há consenso sobre o momento adequado para se realizar a traqueostomia. Além disso, critérios que não sejam relacionados à VM estão sendo utilizados para definir DCC, considerando outros aspectos da síndrome: disfunção múltipla de órgãos, sepse grave, feridas graves. Tais critérios são utilizados para definir DCC em estudos que tratam de economia, a fim de recomendar a quantidade de recursos a serem repassados às unidades hospitalares ${ }^{3,4,8}$.

Um estudo feito em Porto Alegre-RS comparou duas definições de DCC: traqueostomia feita devido à previsão de VMP e duração de VM por mais de 21 dias. Entre os 274 pacientes críticos, 24 eram doentes críticos crônicos (DoCC): 19 pelo critério da traqueostomia, 15 pelo critério da VMP, 10 preenchiam os dois critérios. Os pacientes em VM por mais de 21 dias são mais graves e apresentam maior mortalidade do que os pacientes classificados como DoCC pela traqueostomia 4 .

\section{Epidemiologia}

Apesar das diferenças entre os estudos, é evidente que a incidência de DCC vem aumentando. Estima-se que 5\% a $10 \%$ dos pacientes críticos agudos internados em UTIs se tornem DoCC. A mortalidade da DCC em um ano chega a 48-70\%, maior que a de muitas neoplasias. Além disso, a qualidade de vida dos DoCC que recebem alta é ruim. Apenas $10 \%$ deles vivem com boa qualidade; $70 \%$ necessitam de suporte à vida permanentemente (internação domiciliar ou institucionalização). Até $53 \%$ dos DoCC são liberados da VM, o que ocorre geralmente em até 37 dias de VMP. Os pacientes que não conseguem desmame da VMP em até 60 dias, raramente o farão após esse período ${ }^{1}$ $6,14,17,18,19$

Entre os fatores de risco para DCC, estão: internamento prévio por doença grave, doenças crônicas prévias (principalmente cardíacas, pulmonares ou renais), idade avançada, necessidade de procedimento cirúrgico de grande porte ou em caráter emergencial, índice de massa corporal acima ou abaixo da faixa normal, sepse grave e altos escores de gravidade no momento da admissão à UTI2,18, 20 .

A DCC já é considerada problema de saúde pública para alguns governos, principalmente porque envolve internação prolongada (média de 60 dias, incluindo $20-40 \%$ das diárias de leito das UTIs) e gastos excessivos durante e após a permanência no hospital. Ambientes alternativos para os DoCC estão sendo criados e aprimorados por todo o mundo, a fim de otimizar os recursos e liberar os leitos de UTI para pacientes críticos agudos.
Um desses ambientes são os "long-term acute care hospitals (LTACs)", definidos como hospitais de cuidados agudos com permanência que excede 25 dias $^{1,17,18,19}$.

\section{Fisiopatologia e Quadro Clínico}

É difícil a percepção da transição entre a doença crítica aguda e a síndrome da doença crítica crônica. A DCC não é simplesmente uma extensão temporal da doença crítica aguda, é uma síndrome inflamatória distinta com fenótipo previsível e com planos terapêuticos próprios, que independe do evento inicial (sepse, trauma, cirurgia etc.) ${ }^{18,21}$.

A história "natural" da DCC envolve infecções de repetição (incluindo infecções por fungos e por bactérias multirresistentes a drogas), atelectasia, taquicardia compensatória ao baixo débito cardíaco, desnutrição tipo Kwashiorkor, perda de massa e de força muscular (até diafragmática), hiperglicemia, doença metabólica óssea, deficiência de vitamina $D$, ansiedade (inclusive com transtorno de estresse pós-traumático), transtorno depressivo, alteração do ciclo sono-vigília, déficit cognitivo, coma e delirium, síndrome de imobilidade, lesões por pressão, trombose venosa profunda e embolia pulmonar, hiporexia, constipação, incontinência urinária, urolitíase, polineuropatia e miopatia do doente crítico, hipotensão postural e disfunção do eixo neuroendócrino $2,9,18,21,23,24,25,26,27,28,29,30$.

A fisiopatologia da DCC inclui distúrbios metabólicos, imunológicos, nutricionais e neuroendócrinos, e para entender essas alterações, é necessário conhecer os conceitos de homeostasia e de alostasia. Homeostasia é a habilidade de manter os parâmetros, como temperatura, pressão arterial, $\mathrm{pH}$, frequência cardíaca e respiratória, dentro dos limites normais. Quando o organismo é submetido a um evento estressor, a homeostasia é modulada pelo fenômeno da alostasia: ajuste dos parâmetros fisiológicos para atingir um novo equilíbrio, promovendo estabilidade durante uma mudança ${ }^{18,21}$.

Na doença crítica aguda, o estímulo que leva à alostasia é efetivamente eliminado, e os parâmetros homeostáticos retornam à linha de base. A sobrecarga de estímulos estressantes, por outro lado, pode transformar o benefício da alostasia em consequências fisiopatológicas danosas. Didaticamente, existem quatro estágios fisiopatológicos possíveis para um doente crítico: doença crítica aguda, doença crítica aguda prolongada, doença crítica crônica e recuperação.

A resposta orgânica à doença crítica aguda é marcada pelos hormônios adrenérgicos, estimulados pelo eixo hipotálamohipófise, promovendo catabolismo e priorização de sistemas orgânicos vitais; nesse momento, o objetivo terapêutico é o suporte cardiopulmonar e a correção do insulto agudo, para desativar a alostasia. Se o insulto for grave demais para ser revertido, ocorre a morte. Alternativamente, o insulto pode continuar, diminuir ou se repetir, levando o paciente ao estágio de doença crítica aguda prolongada. Nesse momento, há feedback negativo do eixo hipotálamo-hipófise pelos 
hormônios liberados no estágio anterior, causando diminuição do GH, IGF-1, TSH, T3, T4 e ACTH. Ainda há hipercortisolismo pela produção da adrenal via endotelina-1. Ocorre também hipogonadismo hipogonadotrófico. A alostasia já se torna prejudicial nesse estágio, promovendo disfunções orgânicas persistentes, catabolismo e resistência insulínica. O estágio fisiopatológico de doença crítica crônica começaria em torno do 14 으 dia da doença e da dependência do ventilador mecânico, quando há um estado permanente de sobrecarga alostática. Não há marcador sérico específico para $\mathrm{DCC}^{18,21,31,32,33}$.

\section{Tratamento}

Há poucos estudos sobre o tratamento do DoCC. A terapêutica precisa ser feita por equipe multidisciplinar, incluindo médico clínico, cirurgião, psiquiatra, enfermeiros, nutricionistas, psicólogos, fisioterapeutas, terapeutas ocupacionais, cirurgiõesdentistas e os familiares. Os cuidados são oferecidos durante o internamento e após a alta hospitalar, em diversos ambientes, que incluem do domicílio à UTI, refletindo que a DCC é marcada por episódios de agudização. O DoCC não se beneficia mais das tentativas de reanimação volêmica e da estabilização dos sistemas orgânicos; deve-se objetivar a restauração das reservas fisiológicas, a reabilitação e o desmame da ventilação mecânica ${ }^{18,21}$.

A maioria dos DoCC manifesta alguma alteração psíquica ou neurológica durante o internamento e após a alta hospitalar. Os diagnósticos frequentemente encontrados são: transtorno de ansiedade, transtorno depressivo, déficit cognitivo, delirium, alterações do sono e até dependência química (após longo período em uso de opioides ou sedativos). Um pequeno estudo mostrou que a escolha de sedativos com clonidina em vez de outros sedativos pode reduzir um pouco a taxa de mortalidade na DCC. A identificação dessas condições, sua prevenção e tratamento adequado são importantes para a redução do tempo de internamento e melhora da qualidade de vida ${ }^{10,18,34,}$ 35 .

Um desmame eficiente da VM inclui despertar diário, tentativas diárias de ventilação espontânea e implantação de protocolos organizados para o desmame da VM. Isso previne a VMP e a pneumonia associada à ventilação mecânica, e diminui o tempo de internação na UTI. Pode-se considerar o paciente liberado da VM quando não precisa de suporte ventilatório (exceto por ventilação não invasiva no período noturno) por sete dias ou mais. Vários fatores dificultam o desmame da VMP: desnutrição, neuromiopatia do doente crítico, miopatia por corticoides, broncoaspirações recorrentes, embolia pulmonar, delirium, depressão, ansiedade, privação de sono, infecções, falha em identificar potencial desmame, entre outros ${ }^{6,36}$.

A traqueostomia pode ser feita com segurança à beira do leito e, quando realizada precocemente, pode diminuir o tempo de VM e de internamento na UTI. Prever uma VMP, porém, não é tão simples, e alguns pacientes podem ser submetidos à traqueostomia desnecessariamente. Vale ressaltar que a traqueostomia não altera a mortalidade ${ }^{6,37,38,39}$.

Se o trato gastrointestinal estiver funcionando, a nutrição é preferencialmente feita por via enteral, e uma gastrostomia pode ser instituída se o tempo de sonda enteral ultrapassar 30 dias. A oferta nutricional inclui glutamina, zinco, arginina, vitamina A e vitamina C. Deve-se, inclusive, ter cuidado com as recomendações de oferecer pouca dieta durante a instabilidade clínica dos primeiros dias na UTI, pois maiores quantidades de nutrientes recebidas durante a primeira semana na UTI foram associadas à maior sobrevida e à recuperação física mais rápida, entre os DoCC $12,18,40,41$.

A hiperglicemia é bastante frequente no DoCC, mesmo nos não diabéticos, devido ao estado inflamatório crônico. Devese utilizar insulina para o controle glicêmico, com alvo de até $180 \mathrm{mg} / \mathrm{dL}$ de glicemia capilar. Tal controle é difícil, devido às variações da terapia nutricional, ao uso frequente de corticoides e à imprevisibilidade das alterações hormonais, inclusive com altos níveis de insulinemia endógena em torno do 14으 dia de doença crítica. Para a doença mineral óssea, pode-se ofertar cálcio e vitamina D na dieta, e até bifosfonatos ${ }^{7,18,19,42,43}$.

A etiologia da neuromiopatia do doente crítico é desconhecida, e não há tratamento específico. Precisa-se, portanto, evitar essa condição, controlando os níveis glicêmicos, dando suporte hemodinâmico/ventilatório o mais breve possível durante a fase aguda da doença, utilizando corticoide e bloqueadores neuromusculares de forma restritiva e criteriosa, prescrevendo mobilização e fisioterapia ${ }^{18,29}$.

Os cuidados com as lesões por pressão incluem mobilização, terapia nutricional adequada, avaliação diária da pele localizada em pontos de pressão, tratamento contínuo desde a constatação da categoria um, alívio da pressão sobre as áreas envolvidas, desbridamento da área desvitalizada. A técnica de dar banho nos DoCC também está sendo estudada, a fim de não causar danos, nem dificultar as tentativas de desmame da ventilação mecânica. Na verdade, a mobilização no leito parece ser benéfica em vários sentidos: diminuir o nível sérico de citocinas inflamatórias, facilitando a recuperação clínica; prevenção e tratamento das lesões por pressão e da neuromiopatia do doente crítico $^{18,29,44,45}$.

Quantoàsinfecções de repetiçãoe porbactérias multirresistentes a drogas, deve-se sempre tentar guiar a escolha dos antibióticos por culturas e teste de sensibilidade aos antimicrobianos. Um estudo mostrou que a presença do Mycoplasma pneumoniae no trato respiratório inferior é frequente em pacientes críticos e pode dificultar o desmame da ventilação mecânica. Outro trabalho evidenciou melhora dos quadros de otite média dos DoCC após traqueostomia ${ }^{46,47}$.

Anemia é frequente nos DoCC por vários motivos: perda sanguínea, sepse, disfunção medular, comorbidades. É importante evitar transfusões sanguíneas desnecessárias, pois estão relacionadas a aumento da mortalidade. Em geral, 
pacientes com hemoglobina maior que $7 \mathrm{~g} / \mathrm{dL}$ não precisam de transfusão de concentrado de hemácias ${ }^{48}$.

Para todos os DoCC, os cuidados paliativos são essenciais, incluindo aqueles que estão recebendo tratamento curativo e terapias de suporte de vida. As queixas mais comumente apresentadas são: dor, astenia, sede e boca seca, dispneia, ansiedade e dificuldade de comunicação. Além de aliviar os sintomas, os cuidados paliativos visam à comunicação sobre os objetivos do tratamento com a família e com o próprio paciente (se for possível) e todo o suporte a eles necessário. Já existe um folheto, validado em língua inglesa, contendo informações sobre a DCC para os familiares $4,7,10,11,13,49,50,51,52,53,54,55,69$.

O conhecimento sobre DCC pode fazer o tratamento dos DoCCs ser menos desgastante para os profissionais de saúde, mas talvez o melhor tratamento seja a prevenção ${ }^{12,56}$.

\section{Prognóstico}

Apesar da alta taxa de mortalidade pela DCC, alguns estudos mostraram que tanto os médicos assistentes quanto os familiares têm expectativas superestimadas sobre o desfecho do paciente em um ano. Esse fato decorre da falta de conhecimento sobre a DCC e da deficiência na comunicação da equipe assistencial com os familiares ${ }^{6,20,22}$.

Prognosticar é um desafio para os médicos, e os escores prognósticos geralmente usados para doentes críticos agudos, como o APACHE, não são válidos para o DoCC. Para facilitar a predição de mortalidade, a indicação de cuidados paliativos, a utilização racional de recursos hospitalares e a comunicação sobre DCC, foi criado e validado o escore ProVent (Tabela 1).

Tabela 1. Escore ProVent: variáveis e pontuação

\begin{tabular}{lr}
\hline Variáveis & Pontuação \\
\hline Idade & 0 \\
$<50$ anos & 1 \\
$50-64$ anos & 2 \\
$\geq 65$ & \\
Plaquetas & 0 \\
$>150.000 / \mathrm{mm}^{3}$ & 1 \\
$\leq 150.000 / \mathrm{mm}^{3}$ & \\
Vasopressores & 0 \\
Não & 1 \\
Sim & \\
Hemodiálise & 0 \\
Não & 1 \\
Sim & \\
\hline
\end{tabular}

Adaptado de CARSON, 2012

Com o objetivo de predizer a mortalidade dos DoCC em um ano, esse escore é baseado nos seguintes dados, a serem colhidos no 21ㅇ dia de VM: idade, contagem de plaquetas, necessidade de vasopressores, necessidade de hemodiálise. A pontuação varia de 0 a 5. Para pacientes com escore ProVent $>2$, a mortalidade hospitalar é 43\%; raramente há alta para casa, e a mortalidade em um ano é de 68\% (Tabela 2). Em 2015, um artigo mostrou que o escore também pode ser aplicado precocemente, no 14음 dia de VM; além disso, também indica a mortalidade ainda na UTI. Pacientes com diagnóstico de VMP que conseguiram sair da VM e que, nos 14 dias seguintes, precisaram retornar para o ventilador mecânico também têm maior taxa de mortalidade em um ano $6,20,22,57,58,59,60,61,62,63,64,65$.

Tabela 2. Mortalidade em um ano pelo escore ProVent

\begin{tabular}{lr}
\hline Escore ProVent & Percentual de Mortalidade (IC 95\%) \\
\hline 0 & $20(10-29)$ \\
1 & $36(24-48)$ \\
2 & $56(45-68)$ \\
3 & $81(67-94)$ \\
4 ou 5 & $100(77-100)$ \\
\hline Adaptado de CARSON, 2012 &
\end{tabular}

Adaptado de CARSON, 2012

Pontuações altas no escore Sequential Organ Failure Assessment (SOFA) no 210 dia de VM também podem ser usadas como preditores de mortalidade, 6 meses após a alta hospitalar. $\mathrm{O}$ escore Charlson de comorbidades alto pode ajudar a prognosticar no contexto de DCC, exceto para os pacientes portadores de síndrome de imunodeficiência humana adquirida (SIDA), que pontuam bastante no Charlson, mas têm epidemiologia de DCC semelhante, quando comparados aos sem SIDA ${ }^{63,65,66,67}$

Aparentemente, a mortalidade da DCC após o período de um ano continua a aumentar. Além disso, as taxas de readmissão hospitalar são altas: 40\% em um ano ${ }^{1,12,21,68,70 .}$

\section{CONCLUSÕES}

A doença crítica crônica é uma síndrome de fisiopatologia complexa, caracterizada pela ventilação mecânica prolongada. Sua prevalência é importante, seu impacto socioeconômico é bastante preocupante, e a mortalidade é altíssima. Apesar disso, existem poucos dados sobre a realidade brasileira.

Os profissionais de saúde precisam conhecer melhor essa síndrome, diagnosticá-la e tratá-la adequadamente, inclusive iniciando a abordagem de cuidados paliativos o quanto antes. 


\section{REFERÊNCIAS}

1. Daly BJ, Douglas SL, Gordon NH, Kelley CG, O'Tolle E, Montenegro H, et al. Composite outcomes of chronically critically ill patients 4 months after hospital discharge. Am J Crit Care. 2009 Sep; 18(5):456-64. doi: 10.4037/ajcc2009580. PubMed PMID: 19723866.

2.Loss SH, Marchese CB, Boniatti MM, Wawrzeniak IC, Oliveira RP, Nunes LN, et al. Prediction of chronic critical illness in a general intensive care unit. Rev Assoc Med Bras. 2013 May-Jun; 59(3):241-7. doi: 10.1016/j.ramb.2012.12.002. PubMed PMID: 23680275.

3.Carson SS. Definitions and epidemiology of the chronically critically ill. Respir Care 2012 Jun; 57(6):848-56. doi: 10.4187/respcare.01736. PubMed PMID: 22663962.

4.Boniatti MM, Friedman G, Castilho RK, Vieira SRR, Fialkow L. Characteristics of chronically critically ill patients: comparing two definitions. Clinics 2011 66(4):701-4. doi: 10.1590/S1807-59322011000400027.

5.Kahn JM, Werner RM, David G, Have TRT, Benson NM, Asch DA. Effectiveness of long-term acute care hospitalization in elderly patients with chronic critical illness. Med Care. 2013 Jan; 51(1):4-10. doi: 10.1097/MLR.0b013e31826528a7. PubMed PMID: 22874500.

6.Carson SS, Kahn JM, Hough CL, Seeley EJ, White DB, Douglas IS, et al. A multicenter mortality prediction model for patients receiving prolonged mechanical ventilation. Crit Care Med. 2012 Apr; 40(4):1171-6. doi: 10.1097/ CCM.0b013e3182387d43. PubMed PMID: 22080643.

7.Nelson JE, Mercado AF, Camhi SL, Tandon N, Wallenstei S, August GI, et al. Communication about chronic critical illness. Arch Intern Med 2007 Dec; 167(22):2509-15. doi: 10.1001/archinte.167.22.2509. PubMed PMID: 18071175 .

8.Iwashyna TJ, Hodgson CL, Pilcher D, Orford N, Santamaria JD, Bailey M, Bellomo R. Towards defining persistent critical illness and other varieties of chronic critical illness. Crit Care Resusc. 2015 Sep; 17(3): 215-8. PubMed PMID: 26282262.

9.Wintermann GB, Brunkhorst FM, Petrowski K, Strauss B, Oehmichen F, Poh $M$, Rosendahl J. Stress disorders following prolonged critical illness in survivors of severe sepsis. Crit Care Med 2015 Jun; 43(6): 1213-22. doi: 10.1097/ CCM.0000000000000936. PubMed PMID: 25760659.

10. Hickman RL, Pinto MD. Advance directives lessen the decisional burden of surrogate decision-making for the chronically critically ill. J Clin Nurs 2014 Mar; 23(5-6): 756-65. doi: 10.1111/jocn.12427. PubMed PMID: 24330417.

11.Hickman RL, Daly BJ, Lee E. Decisional conflict and regret: consequences of surrogate decision making for the chronically critically ill. Appl Nurs Res 2012 Nov; 25(4): 271-5. doi: 10.1016/j.apnr.2011.03.003. PubMed PMID: 21658906.

12.Lu HM, Chen L, Wang JD, Hung MC, Lin MS, Yan YH, et al. Outcomes of prolonged mechanic ventilation: a discrimination model based on longitudinal health insurance and death certificate data. BMC Health Serv Res 2012 Apr; 12 100. doi: 10.1186/1472-6963-12-100.

13.Douglas SL, Daly BJ, O'Toole E, Hickman RL. Depression among white and nonwhite caregivers of the chronically critically ill. J Crit Care. 2010 Jun; 25(2): 364. doi: 10.1016/j.jcrc.2009.09.004.

14.Bigatello LM, Stelfox HT, Berra L, Schmidt U, Gettings EM. Outcome of patients undergoing prolonged mechanical ventilation after critical illness. Crit Care Med 2007 Nov; 35(11): 2491-7. doi: 10.1097/01.CCM.0000287589.16724. B2.

15.Van Pelt DC, Milbrandt EB, Qin L, Weissfeld LA, Rotondi AJ, Schulz R, et al. Informal caregiver burden among survivors of prolonged mechanical ventilation. Am J Respir Crit Care Med. 2007 Jan; 175(2): 167-73. doi: 10.1164/

\section{rccm.200604-4930C.}

16.Loss SH, Oliveira RP, Maccari JG, Savi A, Boniatti MM, Hetzel MP, et al. A realidade dos pacientes que necessitam de ventilação mecânica prolongada: um estudo multicêntrico. Rev Bras Ter Intensiva. 2015 Mar; 27(1): 26-35. doi: http://dx.doi.org/10.5935/0103-507X.20150006.

17.Cox CE, Carson SS, Lindquist JH, Olsen MK, Govert JA, Chelluri L, et al. Differences in one-year health outcomes and resource utilization by definition of prolonged mechanical ventilation: a prospective cohort study. Critical Care 2007;11(1): R9. doi: 10.1186/cc5667.

18.Scheinhorn DJ, Hassenpflug MS, Votto JJ, Chao DC, Epstein SK, Doig GS, et al. Ventilator-dependent survivors of catastrophic illness transferred to 23 longterm care hospitals for weaning from prolonged mechanical ventilation. CHEST 2007 Jan; 131(1):76-84. doi: 10.1378/chest.06-1079.

19.Goldwasser R, Farias A, Freitas EE, Saddy F, Amado V, Okamoto VN. Desmame e interrupção da ventilação mecânica. Rev Bras Ter Intensiva. 2007; 19(3):38492.

20.Unroe M, Kahn JM, Carson SS, Govert JA, Martinu T, Sathy SJ, et al. Oneyear trajectories of care and resource utilization for recipients of prolonged mechanical ventilation: a cohort study. Ann Intern Med 2010 Aug; 153(3):16775. doi: 10.7326/0003-4819-153-3-201008030-00007. PubMed PMID: 20679561.

21.Schulman RC, Mechanick Jl. Metabolic and nutrition support in the chronic critical illness syndrome. Respir Care 2012; 57(6):958-77. doi: 10.4187/ respcare.01620. PubMed PMID: 22663970.

22. Carson SS, Garrett J, Hanson LC, Lanier J, Govert J, Brake MC, et al. A prognostic model for one-year mortality in patients requiring prolonged mechanical ventilation. Crit Care Med. 2008 Jul; 36(7):2061-9. doi: 10.1097/ CCM.0b013e31817b8925. PubMed PMID: 18552692.

23. Girard TD. Brain dysfunction in patients with chronic critical illness. Respir Care. 2012 Jun; 57(6):947-55. doi: 10.4187/respcare.01708.

24. Zaborin A, Smith D, Garfield K, Quensen J, Shakhsheer B, Kade M, et al. Membership and behavior of ultra-low-diversity pathogen communities present in the gut of humans during prolonged critical illness. MBio. 2014 Sep; 5(5): e01361-14.

25. Miguélez $M$, Merlani $P$, Gigon F, Verdon M, Annoni JM, Ricou B. Feasibility of a multiple-choice mini mental state examination for chronically critically ill patients. Crit Care Med 2014 Aug; 42(8): 1874-81. doi: 10.1097/ CCM.0000000000000342.

26. Hope AA, Morrison RS, Du Q, Wallenstein S, Nelson JE. Risk factors for longterm brain dysfunction after chronic critical illness. Ann Am Thorac Soc. 2013 Aug; 10(4): 315-23. doi: 10.1513/AnnalsATS.201211-0990C. PubMed PMID: 10.1513/AnnalsATS.201211-0990C.

27. Verceles AC, Lechner EJ, Halpin D, Scharf SM. The association between comorbid illness, colonization status, and acute hospitalization in patients receiving prolonged mechanical ventilation. Respir Care. 2013 Feb; 58(2): 2506. doi: 10.4187/respcare.01677. PubMed PMID: 22709565.

28. Santos PD, Teixeira C, Savi A, Maccari JG, Neres FS, Machado AS, et al. The critical illness polyneuropathy in septic patients with prolonged weaning from mechanical ventilation: is the diaphragm also affected? A pilot study. Respir Care. 2012 Oct; 57(10): 1594-1601. doi: 10.4187/respcare.01396. PubMed PMID: 22417531.

29. Derde S, Hermans G, Derese I, Güiza F, Hedström Y, Wouters PJ, et al. Muscle atrophy and preferential loss of myosin in prolonged critically ill patients. Crit Care Med. 2012 Jan; 40(1): 79-89. doi: 10.1097/CCM.0b013e31822d7c18. 
PubMed PMID: 21926599.

30. Wu JY, Hsu SC, Ku SC, Ho CC, Yu CJ, Yang PC. Adrenal insufficiency in prolonged critical illness. Crit Care. 2008 May; 12(3): R65. doi: 10.1186/cc6895.

31. Bello G, Pennisi MA, Montini L, Silva S, Maviglia R, Cavallaro F, et al. Nonthyroidal illness syndrome and prolonged mechanical ventilation in patients admitted to the ICU. Chest. 2009 Jun; 135(6): 1448-54. doi: 10.1378/ chest.08-1816.

32. Duska F, Fric M, Pazout J, Waldauf $\mathrm{P}$, Tuma P, Pachl J. Frequent intravenous pulses of growth hormone together with alanylglutamine supplementation in prolonged critical illness after multiple trauma: effects on glucose control, plasma IGF-I and glutamine. Growth Horm IGF Res. 2008 Feb; 18(1): 82-7. doi: 10.1016/j.ghir.2007.07.003. PubMed PMID: 17709266.

33. Mebis L, Langouche L, Visser TJ, Van den Berghe G. The type II iodothyronine deiodinase is up-regulated in skeletal muscle during prolonged critical illness J Clin Endocrinol Metab. 2007 Aug; 92(8): 3330-3. doi: 10.1210/jc.2007-0510. PubMed PMID: 17504898.

34. Karir V, Hough CL, Daniel S, Caldwell E, Treggiari MM. Sedation practices in a cohort of critically ill patients receiving prolonged mechanical ventilation. Minerva Anestesiol. 2012 Jul; 78(7): 801-9. PubMed PMID: 22475804.

35. Moritz RD, Machado FO, Pinto EP, Cardoso GS, Nassar SM. Avaliação do uso da clonidina para sedoanalgesia de pacientes sob ventilação mecânica prolongada, internados em unidade de terapia intensiva. Rev Bras Ter Intensiva. 2008 Mar; 20(1): 24-30.

36. Ginz HF, laizzo PA, Urwyler A, Pargger H. Use of non-invasive-stimulated muscle force assessment in long-term critically ill patients: a future standard in the intensive care unit? Acta Anaesthesiol Scand. 2008 Jan; 52(1): 20-7. doi 10.1111/j.1399-6576.2007.01427.x. PubMed PMID: 17714574

37. Zheng Y, Sui F, Chen XK, Zhang GC, Wang XW, Zhao S, et al. Early versus late percutaneous dilational tracheostomy in critically ill patients anticipated requiring prolonged mechanical ventilation. Chin Med J (Engl). 2012 Jun; 125(11): 1925-30. PubMed PMID: 22884055.

38. Clec'h C, Alberti C, Vincent F, Garrouste-Orgeas M, Lassence A, Toledano $D$, et al. Tracheostomy does not improve the outcome of patients requiring prolonged mechanical ventilation: a propensity analysis. Crit Care Med. 2007 Jan; 35(1): 132-8. doi: 10.1097/01.CCM.0000251134.96055.A6.

39. Terra RM, Fernandez A, Bammann RH, Castro ACP, Ishy A, Junqueira JJM. Open bedside tracheostomy: routine procedure for patients under prolonged mechanical ventilation. Clinics (São Paulo). 2007 Aug; 62(4): 427- 32. PubMed PMID: 17823705

40. Wei X, Day AG, Ouellette-Kuntz H, Heyland DK. The association between nutritional adequacy and long-term outcomes in critically III patients requiring prolonged mechanical ventilation: a multicenter cohort study. Crit Care Med. 2015 Aug; 43(8): 1569-79. doi: 10.1097/CCM.0000000000001000.

41. Faisy C, Lerolle N, Dachraoui F, Savard JF, Abboud I, Tadie JM, et al. Impact of energy deficit calculated by a predictive method on outcome in medical patients requiring prolonged acute mechanical ventilation. Br J Nutr. 2009 Apr; 101(7): 1079-8. doi: 10.1017/S0007114508055669. PubMed PMID: 18778528.

42. Via MA, Potenza MV, Hollander J, LiuX, Peng Y, LiJ, etal. Intravenousibandronate acutely reduces bone hyperresorption in chronic critical illness. J Intensive Care Med. 2012 Sep-Oct; 27(5): 312-8. doi: 10.1177/0885066611402156. PubMed PMID: 21436164

43. Duska F, Andel M. Intensive blood glucose control in acute and prolonged critical illness: endogenous secretion contributes more to plasma insulin than exogenous insulin infusion. Metabolism. 2008 May; 57(5): 669-71. doi: 10.1016/j.metabol.2008.01.001. PubMed PMID: 18442631.

44. Happ MB, Tate JA, Swigart VA, DiVirgilio-Thomas D, Hoffman LA. Wash and wean: bathing patients undergoing weaning trials during prolonged mechanical ventilation. Heart Lung. 2010 Nov-Dec; 39(6 Suppl): S47-56. doi: 10.1016/j. hrtlng.2010.03.002.

45. Winkelman C, Higgins PA, Chen YJK, Levine AD. Cytokines in chronically critically ill patients after activity and rest. Biol Res Nurs. 2007 Apr; 8(4): 261-71. doi: 10.1177/1099800406298168. PubMed PMID: 17456587.

46. Muir MT, Cohn SM, Louden C, Kannan TR, Baseman JB. Novel toxin assays implicate Mycoplasma pneumoniae in prolonged ventilator course and hypoxemia. Chest. 2011 Feb; 139(2): 305-10. doi: 10.1378/chest.10-1222. PubMed PMID: 20884727.

47. Chung HK, Lin CC, Wang CY, Lin CD, Tsai MH, Chang CS. Improvement in otitis media with effusion in patients undergoing tracheostomy after prolonged endotracheal intubation. J Otolaryngol Head Neck Surg. 2009 Oct; 38(5):532-6. PubMed PMID: 19769822

48. Zilberberg MD, Stern LS, Wiederkehr DP, Doyle JJ, Shorr AF. Anemia, transfusions and hospital outcomes among critically ill patients on prolonged acute mechanical ventilation: a retrospective cohort study. Crit Care. 2008; 12(2): R60. doi: 10.1186/cc6885.

49. Hickman RL Jr, Daly BJ, Douglas SL, Burant CJ. Evaluating the critical care family satisfaction survey for chronic critical illness. West J Nurs Res. 2012 Apr; 34(3): 377-95. doi: 10.1177/0193945911402522. PubMed Central PMCID: PMC4370175.

50. Carson SS, Vu M, Danis M, Camhi SL, Scheunemann LP, Cox CE, et al. Development and validation of a printed information brochure for families of chronically critically ill patients. Crit Care Med. 2012 Jan; 40(1): 73-8. doi: 10.1097/CCM.0b013e31822d7901. PubMed PMID: 21926610.

51. Choi J, Donahoe MP, Zullo TG, Hoffman LA. Caregivers of the chronically critically ill after discharge from the intensive care unit: six months' experience. Am J Crit Care 2011 Jan; 20(1): 12-22. doi: 10.4037/ajcc2011243. Pubmed PMID: 21196567.

52. Campbell GB, Happ MB. Symptom identification in the chronically critically ill. AACN Adv Crit Care. 2010 Jan-Mar; 21(1): 64-79. doi: 10.1097/ $\mathrm{NCl} .0 \mathrm{~b} 013 \mathrm{e} 3181 \mathrm{c} 932 \mathrm{a} 8$.

53. Camhi SL, Mercado AF, Morrison RS, Du Q, Platt DM, August GI, et al. Deciding in the dark: advance directives and continuation of treatment in chronic critical illness. Crit Care Med. 2009 Mar; 37(3): 919-25. doi: 10.1097/ cCM.0b013e31819613ce.

54. Douglas SL, Daly BJ, Kelley CG, O'Toole E, Montenegro H. Chronically critically ill patients: health-related quality of life and resource use after a disease management intervention. Am J Crit Care. 2007 Sep; 16(5): 447-57. PubMed PMID: 17724242.

55. Happ MB, Swigart VA, Tate JA, Hoffman LA, Arnold RM. Patient involvement in health-related decisions during prolonged critical illness. Res Nurs Health. 2007 Aug; 30(4): 361-72. doi: 10.1002/nur.20197.

56. Roulin MJ, Boul'ch MF, Merlani P. Staff satisfaction between 2 models of care for the chronically critically ill. J Crit Care. 2012 Aug; 27(4): 426.e1-8. doi: 10.1016/j.jcrc.2011.12.019. PubMed PMID: 22421003.

57. Udeh $\mathrm{Cl}$, Hadder $\mathrm{B}$, Udeh $\mathrm{BL}$. Validation and extension of the prolonged mechanical ventilation prognostic model (ProVent) score for predicting 1-year mortality after prolonged mechanical ventilation. Ann Am Thorac Soc. 2015 Dec; 12(12): 1845-51. doi: 10.1513/AnnalsATS.201504-2000C. PubMed PMID: 26418231

58. Boniatti MM, Giustina AD, Marin LG, França J, Santos MC, Vidart J, et al. Mortality in chronically critically ill patients: Expanding the use of the ProVent score. J Crit Care. 2015 Oct; 30(5): 1039-42. doi: 10.1016/j.jcrc.2015.06.022. PubMed PMID: 26169543.

59. Dermot Frengley J, Sansone GR, Shakya K, Kaner RJ. Prolonged mechanical 
99 Revisão sobre doença crítica crônica

ventilation in 540 seriously ill older adults: effects of increasing age on clinical outcomes and survival. J Am Geriatr Soc. 2014 Jan; 62(1): 1-9. doi: 10.1111/ jgs.12597. PubMed PMID: 24404850.

60. Santana-Cabrera L, Lorenzo-Torrent R, Sánchez-Palacios M, Martín Santana $J D$, Hernández JRH. Influence of age in the duration of the stay and mortality of patients who remain in an Intensive Care Unit for a prolonged time. Rev Clin Esp (Barc) 2014 Mar; 214(2): 74-8

61. Tu ML, Tseng CW, Tsai YC, Wang CC, Tseng CC, Lin MC, et al. Reinstitution of mechanical ventilation within 14 days as a poor predictor in prolonged mechanical ventilation patients following successful weaning. Scientific World Journal. 2012; 2012: 957126. doi: 10.1100/2012/957126. PubMed PMID: 22924030.

62. Douglas SL, Daly BJ, O’Toole EE, Kelley CG, Montenegro H. Age differences in survival outcomes and resource use for chronically critically ill patients. J Crit Care. 2009 Jun; 24(2): 302-10. doi: 10.1016/j.jcrc.2008.02.004. PubMed Central PMCID: PMC2796433.

63. Moreau D, Timsit JF, Vesin A, Garrouste-Orgeas M, de Lassence A, Zahar $J R$, Adrie C, Vincent F, Cohen Y, Schlemmer B, Azoulay E. Platelet count decline: an early prognostic marker in critically ill patients with prolonged ICU stays. Chest 2007 Jun; 131(6): 1735-41. doi: 10.1378/chest.06-2233. PubMed: PMID 17475637.

64. Verceles AC, Weiler B, Koldobskiy D, Goldberg AP, Netzer GS, John D. Association Between Vitamin D Status and Weaning From Prolonged Mechanical Ventilation in Survivors of Critical Illness. Respir Care. 2015 Jul; 60(7): 1033-9. doi: 10.4187/respcare.03137. PubMed PMID: 25715347.

65. Piotto RF, Ferreira FB, Colósimo FC, Silva GS, Sousa AG, Braile DM. Fatores preditores independentes de ventilação mecânica prolongada em pacientes submetidos à cirurgia de revascularização miocárdica. Br J Cardiovasc Surg. 2012 Dez; 27(4): 520-8. doi: http://dx.doi.org/10.5935/1678-9741.20120093.

66. Frengley JD, Sansone GR, Uppal K, Vecchione JJ, Kaner RJ. A comparison of seriously ill patients with or without AIDS requiring prolonged mechanical ventilation. J Crit Care. 2012 Dec; 27(6): 594-601. doi: 10.1016/j. jcrc.2012.05.010. PubMed PMID: 22762929.

67. Lee K, Hong SB, Lim CM, Koh Y. Sequential organ failure assessment score and comorbidity: valuable prognostic indicators in chronically critically ill patients. Anaesth Intensive Care. 2008 Jul; 36(4): 528-34. PubMed PMID: 18714621.

68. Rose L, Fraser IM. Patient characteristics and outcomes of a provincial prolonged-ventilation weaning centre: a retrospective cohort study. Can Respir J. 2012 May-Jun; 19(3): 216-20. PubMed PMID: 22679615.

69. Nelson JE, Hope AA. Integration of palliative care in chronic critical illness management. Respir Care. 2012 Jun; 57(6):1004 -12. doi: 10.4187/ respcare.01624. PubMed PMID: 22663973.

70. Hartl WH, Wolf $\mathrm{H}$, Schneider $\mathrm{CP}$, Küchenhoff $\mathrm{H}$, Jauch KW. Acute and long-term survival in chronically critically ill surgical patients: a retrospective observational study. Crit Care. 2007; 11(3): R55. doi: 10.1186/cc5915. PubMed Central PMCID: PMC2206407.

\section{Como citar este artigo/How to cite this article:}

Leitão SM, Wirtzbiki PM, Oliveira OJN. Doença crítica crônica: artigo de revisão narrativa. J Health Biol Sci. 2018 Jan-Mar; 6(1): $92-99$. 